\title{
Mash1 and neurogenin1 Expression Patterns Define Complementary Domains of Neuroepithelium in the Developing CNS and Are Correlated with Regions Expressing Notch Ligands
}

\author{
Qiufu Ma, ${ }^{1}$ Lukas Sommer, ${ }^{1}$ Peter Cserjesi, ${ }^{2}$ and David J. Anderson ${ }^{1}$ \\ ${ }^{1}$ Division of Biology 216-76, Howard Hughes Medical Institute, California Institute of Technology, Pasadena, California \\ 91125, and 2Department of Anatomy and Cell Biology, College of Physicians and Surgeons of Columbia University, \\ New York, New York 10032
}

\begin{abstract}
Genetic studies in Drosophila and in vertebrates have implicated basic helix-loop-helix (bHLH) genes in neuronal fate determination and cell type specification. We have compared directly the expression of Mash1 and neurogenin1 (ngn1), two bHLH genes that are expressed specifically at early stages of neurogenesis. In the PNS these genes are expressed in complementary autonomic and sensory lineages. In the CNS in situ hybridization to serial sections and double-labeling experiments indicate that Mash1 and ngn1 are expressed in adjacent and nonoverlapping regions of the neuroepithelium that correspond to future functionally distinct areas of the brain. We also showed that in the PNS several other bHLH genes exhibit
\end{abstract}

similar lineal restriction, as do ngn 1 and Mash1, suggesting that complementary cascades of bHLH factors are involved in PNS development. Finally, we found that there is a close association between expression of $n g n 1$ and Mash1 and that of two Notch ligands. These observations suggest a basic plan for vertebrate neurogenesis whereby regionalization of the neuroepithelium is followed by activation of a relatively small number of bHLH genes, which are used repeatedly in complementary domains to promote neural determination and differentiation.

Key words: bHLH proteins; Mash1; neurogenins; Notch ligands; neurogenesis; cell lineage
Central questions in developmental neurobiology include understanding how the nervous system is patterned into functionally distinct areas and how cellular diversity is generated within these areas. These questions have been addressed, using genetic analysis in the fruit fly Drosophila. Several important concepts have emerged from this system. First, proneural genes such as achaetescute (ac-sc) and atonal, which encode transcription factors of the basic helix-loop-helix (bHLH) type, function as neural determination genes (Jan and Jan, 1993) (for review, see Weintraub, 1993). Second, distinct proneural genes are used in different neuronal sublineages. For example, $a c-s c$ is essential for external sensory organ formation, whereas atonal is required for that of chordotonal organs (Jan and Jan, 1994). Moreover, ectopic expression of $a s$-sc in the wing disk promotes external sensory organ formation (Rodriguez et al., 1990; Brand et al., 1993), whereas that of atonal promotes chordotonal but not external organ formation (Jarman et al., 1993). Thus, proneural genes play a critical role not only in the choice of neural fate but also in the choice of neuronal subtype.

The functional characterization of vertebrate homologs of Drosophila proneural genes has suggested that the principles described above may be applicable to mammalian neurogenesis as

\footnotetext{
Received Dec. 20, 1996; revised Feb. 19, 1997; accepted March 6, 1997.

Q.M. and L.S. are Associates of the Howard Hughes Medical Institute; D.J.A. is an Investigator of the Howard Hughes Medical Institute. We are grateful to Gerry Weinmaster for providing the Jagged probe, to Domingos Henrique for the mouse Dll-1 probe, to Richard Baer for the mouse NSCL1 probe, to Eric Olson and two anonymous reviewers for their helpful comments, and to Tetsu Saito for the doublelabel in situ hybridization protocol.

Correspondence should be addressed to Dr. David J. Anderson at the above address.

Copyright (C) 1997 Society for Neuroscience 0270-6474/97/173644-09\$05.00/0
}

well. For example, the mammalian ac-sc homolog 1 (Mash1) (Johnson et al., 1990) is expressed in subsets of neuronal precursors in both the PNS and CNS (Lo et al., 1991; Guillemot and Joyner, 1993; Guillemot et al., 1993). Within the PNS Mash1 expression is restricted to autonomic progenitors (Lo et al., 1991). More recently, we have shown that a novel atonal-related gene, neurogenin (now called ngn1), is, conversely, expressed in sensory but not autonomic ganglia in the PNS (Ma et al., 1996). Thus, just as the proneural genes $a c-s c$ and ato define distinct neuronal sublineages in Drosophila, Mash1, and ngn1 define two major neuronal sublineages within the mammalian PNS.

To obtain further insights into the functions of Mash1 and ngn1 in the CNS as well as the PNS, we have compared directly the expression patterns of ngnl, Mash1, and several other genes by single-color or double-color in situ hybridization to adjacent sections. We find that in many embryonic CNS regions ngnl and Mash1 are expressed in complementary domains that exhibit adjacent and nonoverlapping boundaries, suggesting that they are involved in the development of distinct neuronal populations. Within the PNS, moreover, lineages expressing either ngn1 or Mash1 subsequently express other bHLH genes, such as NSCL1 and $e H A N D$ with similar lineal restrictions, suggesting that different classes of peripheral neurons may be specified by complementary cascades of bHLH proteins. Finally, in the CNS, expression of ngn1 and Mash1 is associated closely with that of two vertebrate Notch ligands, Delta-1 (Dll-1) (Bettenhausen et al., 1995) and/or Jagged (Lindsell et al., 1995). This overlap is consistent with a potential functional interaction between these two sets of proteins during neuronal fate determination, as demonstrated for their homologs in Drosophila and Xenopus (Ghysen et al., 1993; Chitnis and Kintner, 1996; Ma et al., 1996). 


\section{NEUROGENIN1}
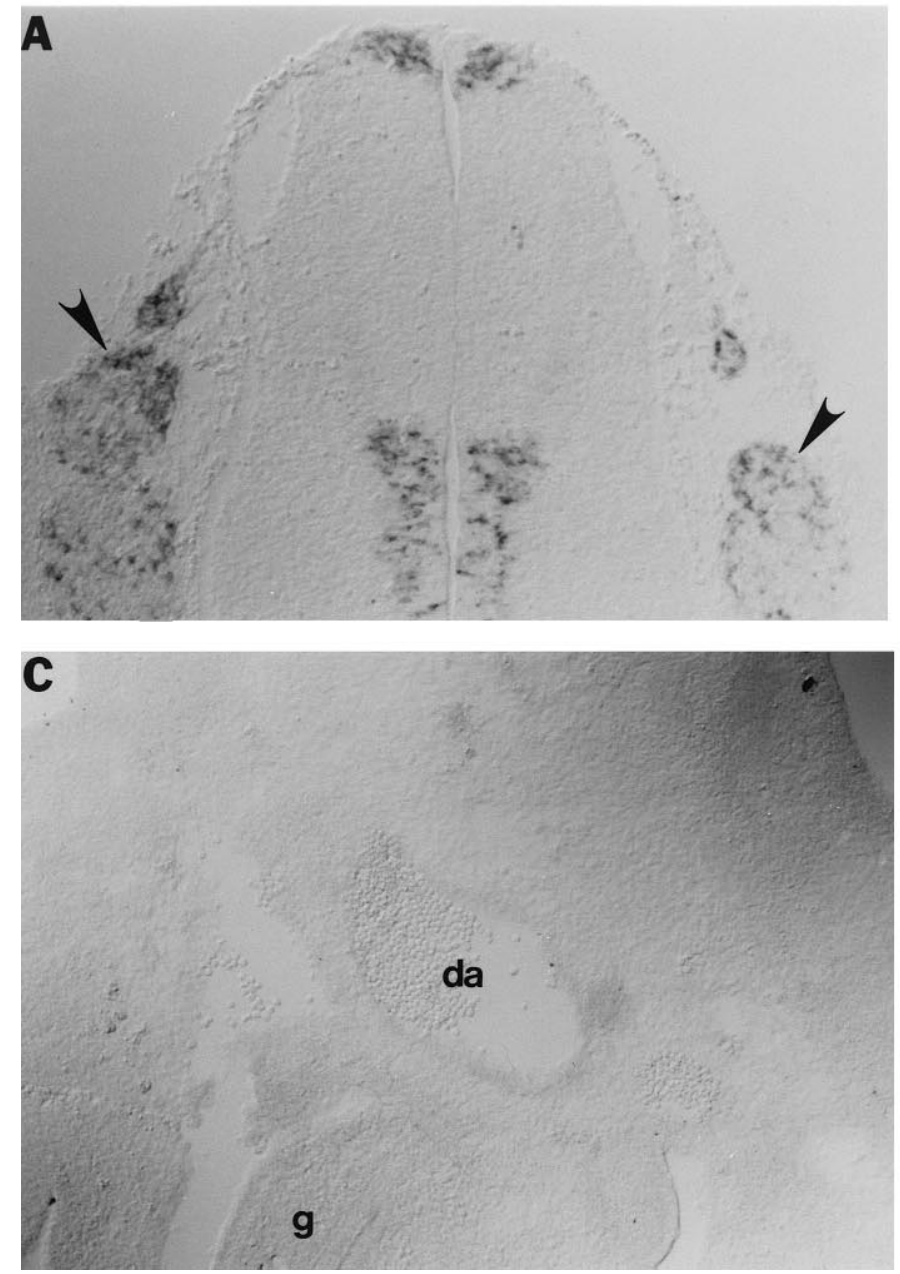

\section{MASH1}
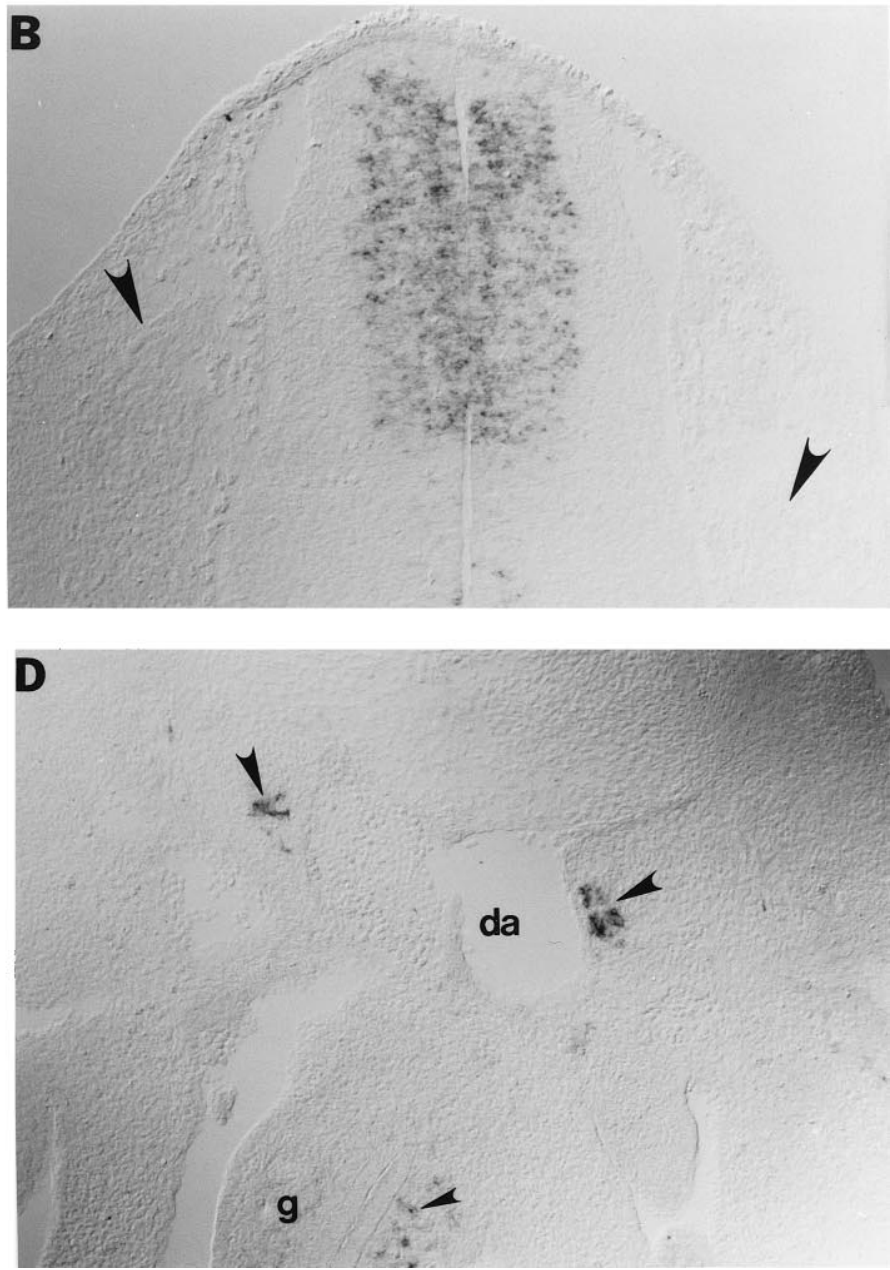

Figure 1. Complementary expression of ngn1 and Mash1 during development of the PNS. Shown are adjacent transverse sections through the trunk region of E13.5 $(A, B)$ or E12.5 $(C, D)$ rat embryos. ngn1 is expressed in DRG $(A$, arrowhead $)$ in which Mash1 is not expressed (B, arrowhead ). Conversely, Mash1 is expressed in sympathetic ganglia adjacent to the dorsal aorta $(d a)(D$, large arrowheads) and enteric ganglia (gut, $g)(D$, small arrowhead), none of which express ngn1 $(C)$. Mutual exclusivity between Mash1 and ngn1 also is observed in the ventricular zone of the spinal cord ( $A$, $B$; see also Fig. 4 ).

\section{MATERIALS AND METHODS}

In situ hybridization. Digoxigenin-labeled in situ hybridization was performed as previously described (Groves et al., 1994) with the following probes: ngn1 (Ma et al., 1996), Mash1 (Lo et al., 1991), Dll-1 (from Domingos Henrique), Jagged (Lindsell et al., 1995), eHAND (Cserjesi et al., 1995), and NSCL1 (Begley et al., 1992). For double-color in situ hybridization $n g n 1$ and Dll-1 were labeled with digoxigenin-UTP (Boehringer Mannheim, Indianapolis, IN), whereas Mash1 and Jagged were labeled with fluorescein-UTP (Boehringer Mannheim). A detailed procedure is available on request.

\section{RESULTS}

\section{Complementary cascades of bHLH gene expression in the PNS}

Previously, we reported in separate studies that MASH1 protein is expressed in peripheral autonomic but not sensory ganglia (Lo et al., 1991) and that ngn1 mRNA is expressed, conversely, in early sensory but not autonomic ganglia (Ma et al., 1996). To confirm this apparent mutual exclusivity of expression in the PNS, we performed a series of in situ hybridization experiments with Mash1 and ngnl probes on adjacent serial sections of rat embryos of various ages. At embryonic day (E) 13.5, ngn1 mRNA was detected in a subset of cells in the dorsomedial margins of the DRG
(Fig. 1A, arrowheads), whereas no Mash1 transcripts were detected in this region (Fig. $1 B$, arrowheads). Conversely, at E12.5 Mash1 mRNA was detected easily in forming sympathetic and enteric ganglia (Fig. 1D, large and small arrowheads, respectively), whereas no ngn 1 mRNA was detectable in this region on adjacent sections (Fig. 1C). Similar observations were made in embryos of other ages (data not shown), indicating that this apparent reciprocity is not a function of developmental stage. The mutual exclusivity of expression in the PNS extended to cranial sensory ganglia; ngn1 mRNA was detected in trigeminal ganglia (Fig. $2 C$, $T G$ ), the otic epithelium (small arrow), and the acoustic component of the facio-acoustic complex (large arrow), whereas none of these structures expressed Mash1 (Fig. 2D).

Several other bHLH genes have been isolated that exhibit complementary expression in the PNS similar to that observed for Mash1 and ngn1. For example, NSCL1 and NSCL2, both related to the lymphoid-specific gene $S C L$ (Porcher et al., 1996), were reported to be expressed in sensory but not autonomic ganglia (Begley et al., 1992; Göbel et al., 1992), as was NeuroD (Lee et al., 1995); $e H A N D /$ Thing1/Hxt (hereafter referred to as $e H A N D)$ was, conversely, reported to be expressed in autonomic but not sensory 


\section{NEUROGENIN1}
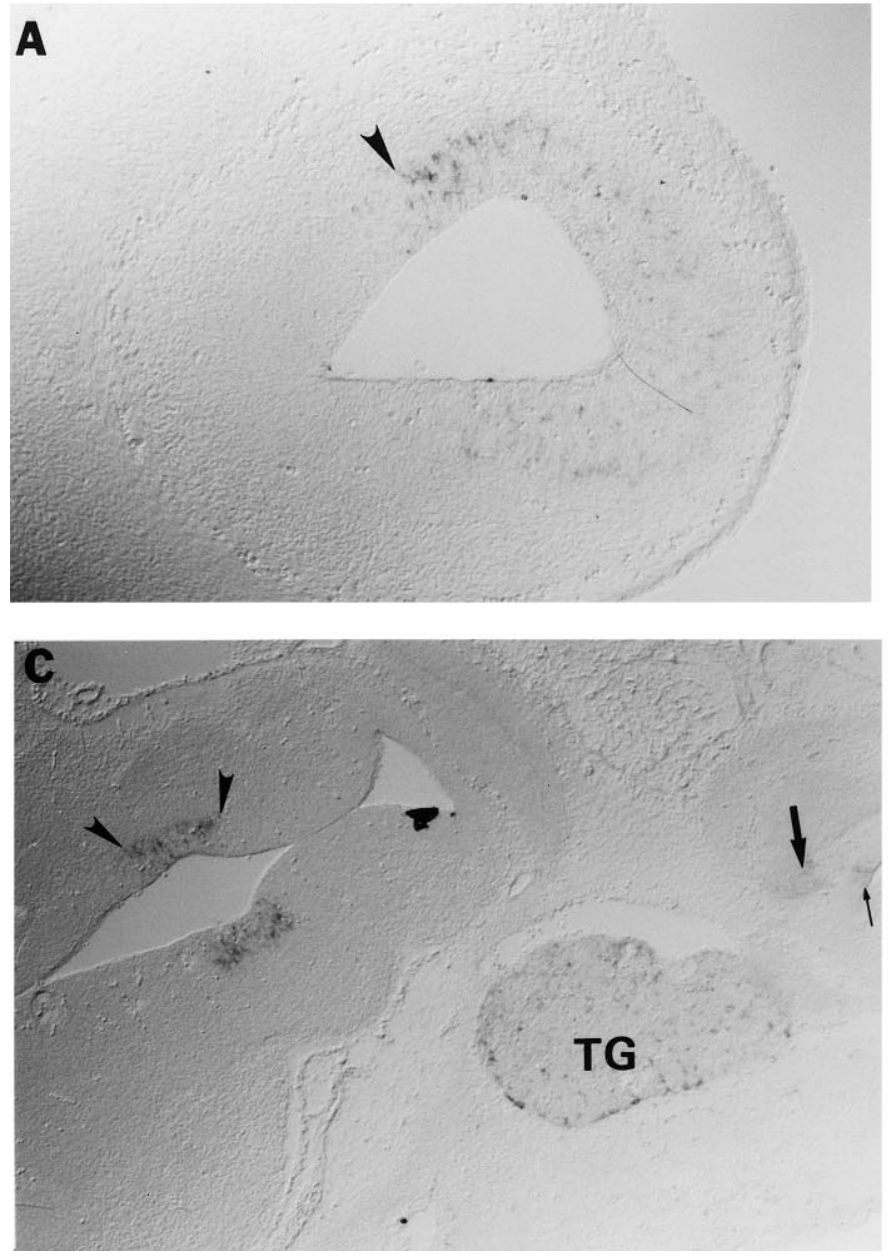

MASH1
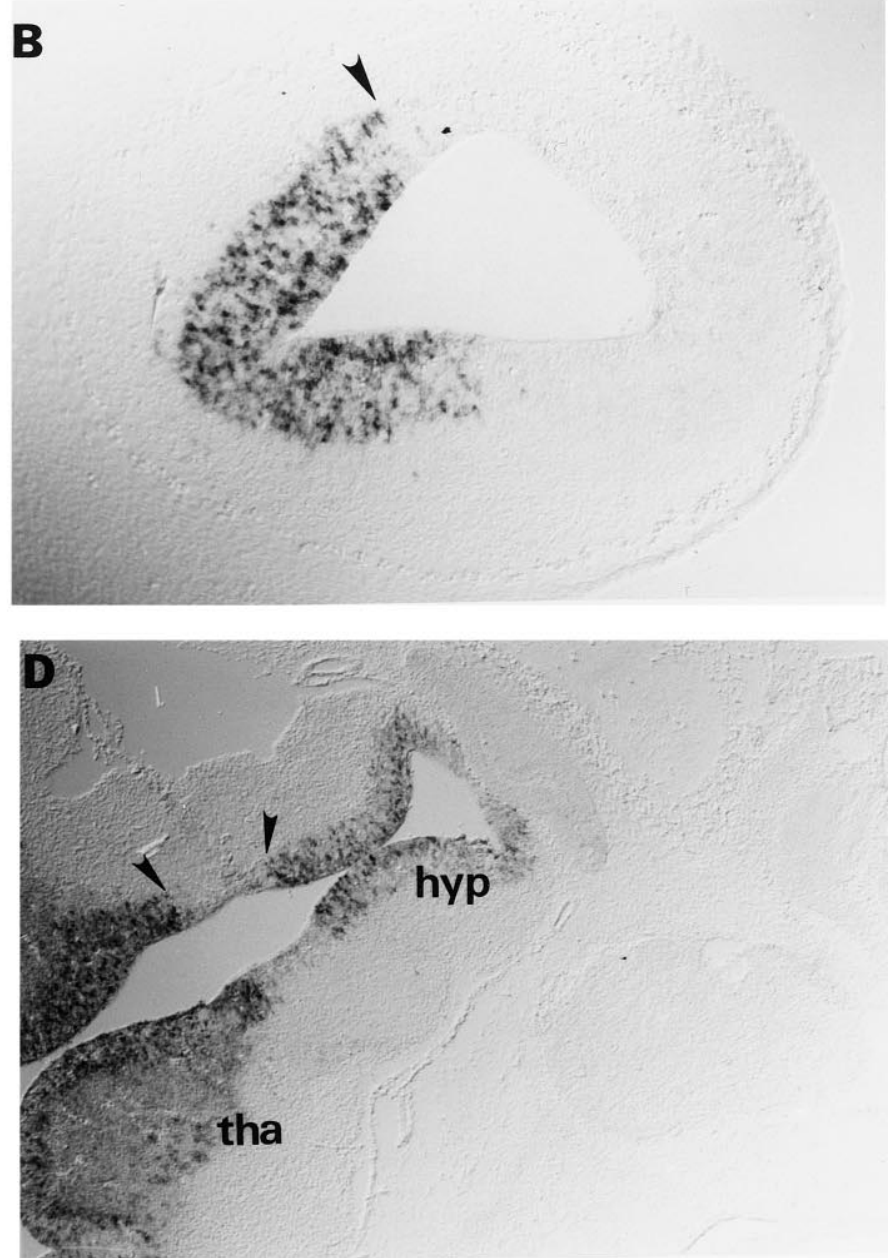

Figure 2. Mutual exclusivity of ngn1 and Mash1 expression in forebrain and cranial sensory ganglia. Transverse sections through the developing olfactory lobe $(A, B$, in high magnification) and the diencephalon $(C, D)$ of an E14.5 rat embryo. ngn1 is expressed in the dorsal/rostral side of the olfactory lobe $(A)$, whereas Mash1 is expressed in the ventral/caudal side $(B)$. Mash1 is expressed throughout the hypothalamus (hyp) and the thalamus $(t h a)$ except for a narrow region $(D)$ in which $n g n 1$ is expressed $(C$, arrowheads). ngn1 is expressed in the trigeminal ganglion $(C$, TG), the acoustic component of the facio-acoustic complex $(C$, large arrow), and the otic epithelium $(C$, small arrow), none of which express Mash1 $(D)$. Expression of neurogenin1 in these cranial ganglia is much stronger in younger embryos (Sommer et al., 1996).

ganglia (as well as in smooth muscle) (Cross et al., 1995; Cserjesi et al., 1995; Hollenberg et al., 1995). These observations raised the question of the temporal relationship between expression of these other bHLH genes and that of Mash1 and ngnl.

In trunk sensory ganglia early expression of both ngn1 and NSCL1 expression is detected at E11.5 (Fig. 3A,B, arrowheads). However, at this age, expression of the former gene is stronger and is detected in more cells than that of the latter (compare Fig. $3 A, B)$. Conversely, at E12.5 expression of $n g n 1$ has begun to decline (Fig. 3C, arrowheads), whereas that of NSCL1 has increased (Fig. 3D, arrowheads). These data suggest that expression of NSCL1 follows or persists longer than that of ngn1. In support of the former interpretation, $n g n 1$ mRNA at E12.5 is expressed most strongly in cells at the dorsomedial margins of the ganglia in which precursors are located; conversely, NSCL1 mRNA is expressed strongly in interior regions of the ganglia in which differentiated neurons are present (compare Fig. 3D). These data suggest that ngn1 expression precedes that of NSCL1 during sensory neurogenesis. In the CNS, ngn1 expression also precedes that of NeuroD (Ma et al., 1996; Sommer et al., 1996). In the PNS, however, we did not observe a temporal difference between $n g n 1$ and NeuroD expression during early stages of DRG development (data not shown). Therefore, NSCL1 seems to be the latest expressed of the three bHLH genes we examined during sensory neurogenesis.

In the case of autonomic ganglia, we wished to know the relationship between the expression of $e H A N D$ and that of Mash1. However, we were unable to detect any temporal separation between the expression of these two bHLH genes in sympathetic ganglia (Fig. 3E; data not shown). Therefore, to ask whether expression of $e H A N D$ is dependent on Mash1 function, we examined its expression in Mash1 knock-out mice (Guillemot et al., 1993). No $e H A N D$ mRNA was detected in the sympathetic anlagen of these mutant embryos (Fig. $3 F$ ). This does not simply reflect an absence of neural crest-derived cells from these ganglion primordia, however, because we have shown previously that immature sympathetic neuron precursors are present in such mutant embryos (Sommer et al., 1995). These data, therefore, indicate that in autonomic ganglia $e H A N D$ expression requires that of Mash1. This, in turn, suggests that $e H A N D$ is expressed 


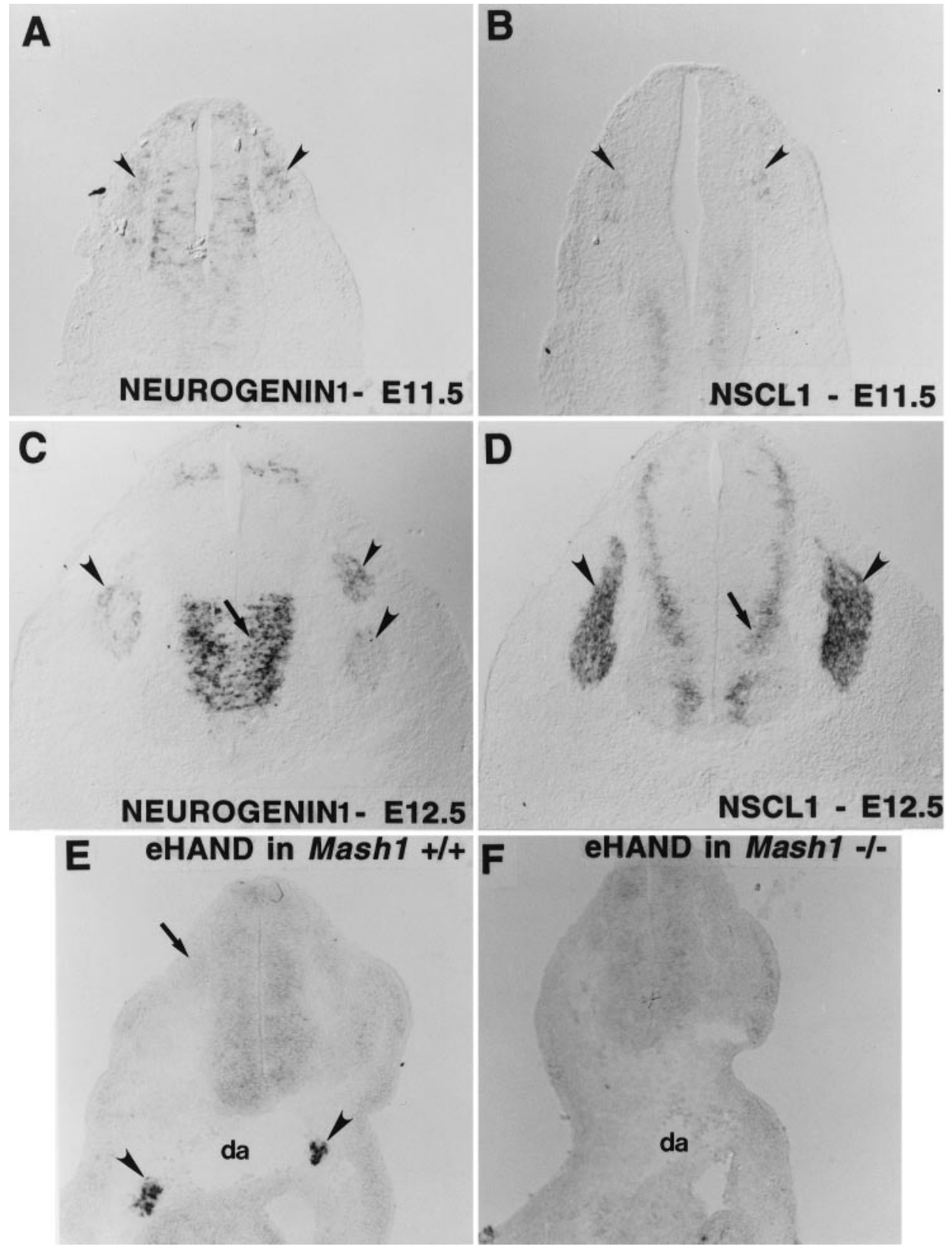

Figure 3. Lineage-specific bHLH cascades within the PNS. Both ngn1 and NSCL1 are expressed in rat DRG at E11.5 and E12.5 ( $A-D$, arrowheads). NSCL1 expression is relatively weak at E11.5 ( $B$, arrowheads) but strong at E12.5 (D, arrowheads). ngn1 expression at E12.5 has begun to fade in the ventral half of the DRG ( $C$, arrowheads). Expression of NSCL1 is displaced more laterally from the ventricular zone $(D$, arrow) than that of $n g n 1$ $(C$, arrow). eHAND is expressed in the sympathetic ganglia of E10.5 mouse embryo $(E$, arrowheads) but not in the sensory ganglia $(E$, arrows). Expression of $e H A N D$ in the sympathetic ganglia is undetectable in Mash1 knock-out mice of similar age $(F)$. either downstream of, or cross-regulated by, MASH1. However, Mash1 expression is not sufficient for expression of $e H A N D$, as seen in the dorsal spinal cord where Mash1, but not $e H A N D$, is expressed (compare Figs. 1B, 3E).

\section{Mutually exclusive domains of Mash1 and ngn1 expression in the CNS}

The foregoing observations of mutually exclusive bHLH gene expression in the PNS raised the question of whether this was true in the CNS as well. As detailed below, the results indicate that expression of Mash1 and ngnl subdivides many regions of the ventricular zone into adjacent and nonoverlapping domains. At E13.5, for example, ngn1 is expressed in two zones that run the length of the spinal cord, a thin one close to the roof plate and a broad one in the ventral half (Fig. 1A). Mash1, conversely, appears to be expressed between these two ngnl stripes (Fig. 1B). Double-label in situ hybridization to sagittal sections (passing through the ventricular zone of the spinal cord) indicated that the boundaries of Mash1 and ngnl expression are adjacent and nonoverlapping (Fig. 4B). The combined expression of Mash1 and ngnl accounts for essentially the entire ventricular zone of the spinal cord, with the exception of a small dorsal region in which Math1 is expressed (Akazawa et al., 1995) and the floorplate.

Similarly, in the telencephalon, Mash1 is expressed in the presumptive basal ganglia (Fig. $4 A$, str), whereas $n g n 1$ is expressed in the presumptive cortex (ctx), as is the related gene ngn2 (Gradwohl et al., 1996; Sommer et al., 1996). Double labeling again revealed that the boundaries between the telencephalic expression domains of $n g n 1$ and Mash1 are adjacent and nonoverlapping (Fig. 4A, arrowheads). A similar mutual exclusivity was observed elsewhere in the forebrain. For example, within the prospective olfactory lobes, ngnl is expressed in the dorsal part (Fig. 2A), whereas Mash1 mRNA is detected in the ventral regions (Fig. $2 B$ ). In the diencephalon Mash1 is expressed throughout the ventricular zone, with the exception of a small region (Fig. 2D, 


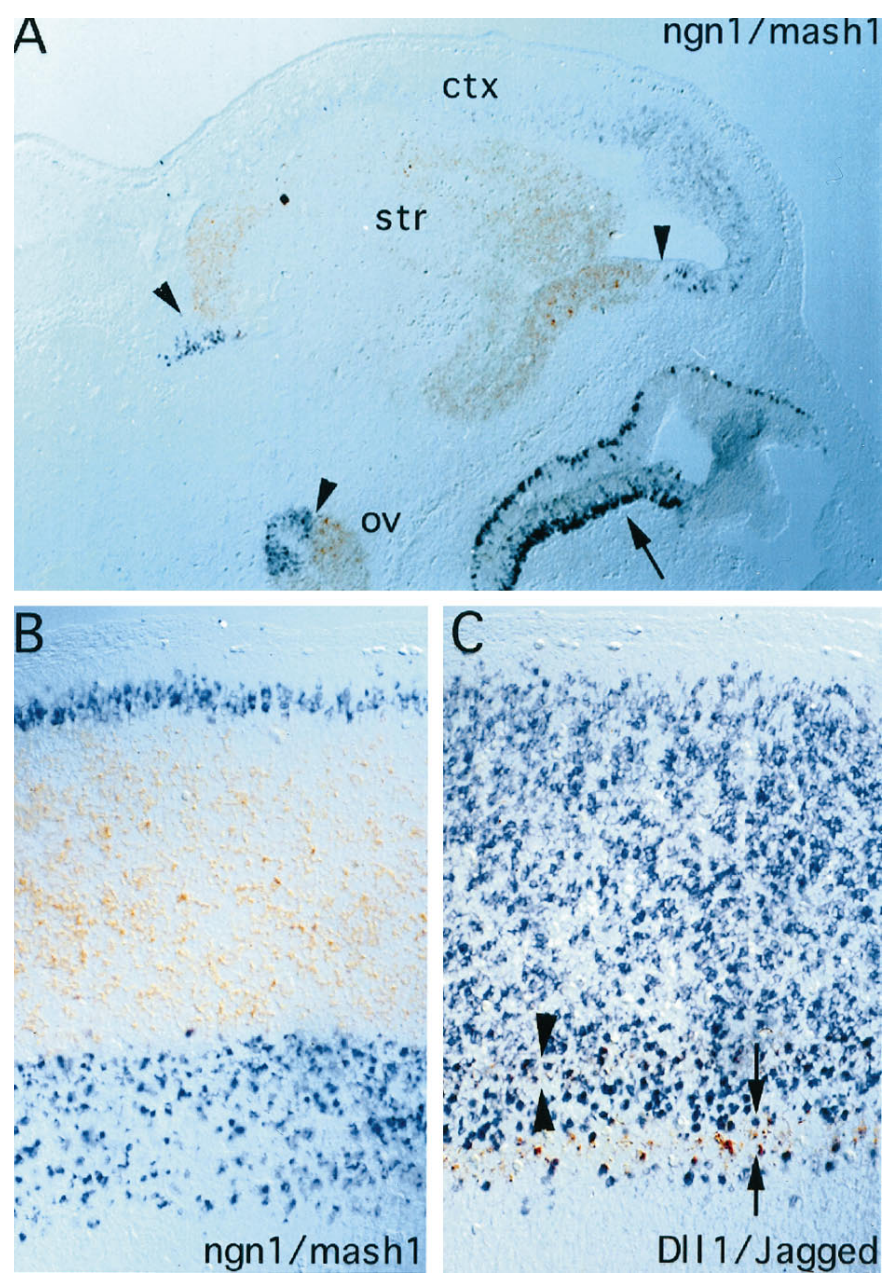

Figure 4. Mutually exclusive expression of Mash1 and ngn1 in spinal cord and forebrain of E14.5 rat embryos demonstrated by double-label in situ hybridization. Shown are sagittal sections of forebrain $(A)$ and spinal cord $(B)$; rostral is to the right, and dorsal is to the top in $A$ and $B$. $B$, The section was cut through the ventricular zone, just lateral to the central canal. ngn1 expression is visualized with the purple chromogen and Mash1 expression with the brown. A, The borders between ngn1 and Mash1 expression are indicated by arrowheads. ngn 1 is expressed in the presumptive cortex (ctx), and Mash1 is expressed in the striatum (str). Complementarity also is seen in optic vesicle (ov). Mash1 and ngn1 are expressed in a partially overlapping manner in the olfactory epithelium (arrow; see also Fig. 5). B, The boundaries between Mash1 and ngn1 expression are adjacent and nonoverlapping. $C$, Sagittal section through spinal cord adjacent to that shown in $B$, double-labeled with probes for Jagged (brown) and Dll-1 (purple). Double arrowheads demarcate the top stripe of Jagged mRNA expression (compare Fig. 6B, arrow), and double arrows demarcate the bottom stripe (compare Fig. 6B, arrowhead). The top stripe of Jagged mRNA (double arrowheads) partially overlaps the ventral boundary of the major dorsal Dll-1 expression domain (compare Fig. $6 D$, arrow), although it is not possible to determine at this level of resolution whether individual cells coexpress both genes. The ventral stripe of Jagged mRNA (compare Fig. $6 B$, arrowhead) appears perfectly interdigitated between the two stripes of Dll-1 mRNA (compare Fig. 6D, arrow and arrowhead). Note that the boundaries of Jagged and Dll-1 do not correspond to those of Mash1 and $n g n 1$; however, Jagged is expressed only in the ngn1-expressing region (see also Fig. 6).

arrowheads) in which ngn1 is expressed instead (Fig. 2C). Such mutual exclusivity also is seen within the optic vesicle (Fig. $4 A$, arrowhead and ov).

In addition to this striking complementarity of Mash1 and ngn1 expression, we also observed some CNS regions that express both genes, including the olfactory epithelium (Fig. $5 A, B$ ), midbrain (Fig. $5 C, D)$, and (at early stages (E11.5-E12.5)) the ventral spinal cord (data not shown). Within these regions of overlap, differences in the expression pattern of the two genes are, nevertheless, evident. For example, in the E14.5 olfactory epithelium, ngn1 expression is restricted to cells located in the basal layer (Fig. 5A), whereas Mash1 is expressed in cells that span the epithelium (Fig. $5 B$ ). In the midbrain the domain of highest ngnl expression appears displaced laterally from the ventricular wall, whereas that of Mash1 fills the entire ventricular zone (compare Fig. 5C,D). Moreover, expression of Mash1 appears relatively uniform (Fig. $5 D$ ), whereas that of $n g n 1$ is restricted to a subset of cells (Fig. $5 C$ ).

\section{Relationship of Mash1 and ngn1 expression to that of Delta and Jagged}

In Drosophila the proneural genes $a c$-sc both activate expression of the lateral inhibitory signal Delta and are, in turn, repressed by signaling through Notch, a receptor for Delta (Ghysen et al., 1993). Similarly, in Xenopus X-ngnr-1 activates expression of $X$-Delta- 1 and is negatively regulated by signaling through X-Notch-1 (Ma et al., 1996). Recently, two mammalian Notch ligands, Dll-1 and Jagged, have been identified (Bettenhausen et al., 1995; Lindsell et al., 1995). To determine whether MASH1 and/or Neurogenin1 similarly might activate expression of these Notch ligands in mammals, we compared their expression with that of Dll-1 and Jagged.

In the spinal cord at E13.5, Jagged is expressed primarily in the ventral region in several narrow longitudinal (i.e., rostrocaudal) stripes that appear as bands across the ventricular zone in transverse sections (Fig. 6B, arrow; see also Lindsell et al., 1995). Comparison of adjacent sections indicates that these bands lie within the region in which $n g n 1$ also is expressed (compare Fig. $6 A, B$; see also Fig. $4 B, C)$. Moreover, the top band of Jagged mRNA expression (Fig. 6B, arrow) appears well correlated with the dorsal boundary of the band of ngnl expression (Fig. $6 A$, arrow). In contrast to Jagged, Dll-1 is expressed primarily in the prospective alar (dorsal) region of the spinal cord (Fig. 6D; see also Bettenhausen et al., 1995; Lindsell et al., 1996) in a domain that encompasses the entire band of Mash1 expression (Figs. 4C, $6 C$ ). Double-label in situ hybridization confirms that some boundaries of Jagged and Dll-1 expression, like those of Mash1 and ngn1, are mutually exclusive (Fig. 4C, arrows), confirming previous observations made in rat (Lindsell et al., 1996) and chick (Myat et al., 1996). However, the dorsal stripe of Jagged expression (Fig. $6 B$, arrow) overlaps the ventral boundary of $D l l-1$ expression (Fig. $6 D$, arrow), as confirmed by double-labeling on sagittal sections (Fig. $4 C$, arrowheads), so that the complementarity of Notch ligand expression in the spinal cord is not perfect.

Although both Mash1 and ngn1 and Jagged and Dll-1 exhibit complementary boundaries of expression in the spinal cord, comparison of adjacent sections indicates that these boundaries are located in distinct regions. For example, Dll-1 expression (Fig. $6 D$ ) extends dorsally past the top boundary of the Mash1 band (Fig. $6 C$ ) to encompass the thin stripe of ngn 1 expression adjacent to the roof plate (Fig. 6A, arrowhead; compare Fig. $4 B$ vs $C$ ). Furthermore, the ventral boundary of $D l l-1$ expression (Fig. 6D, arrow) overlaps the top part of the ventral band of $n g n l$ expression (Fig. 6A, arrow). Thus, expression of Dll-1 is detected in both Mash1- and ngn1-expressing regions. Interestingly, however, Jagged expression is detected only in regions that express ngn1 (compare Fig. $6 A$ vs $B$ ).

The correlation between Jagged and ngn1 expression observed 

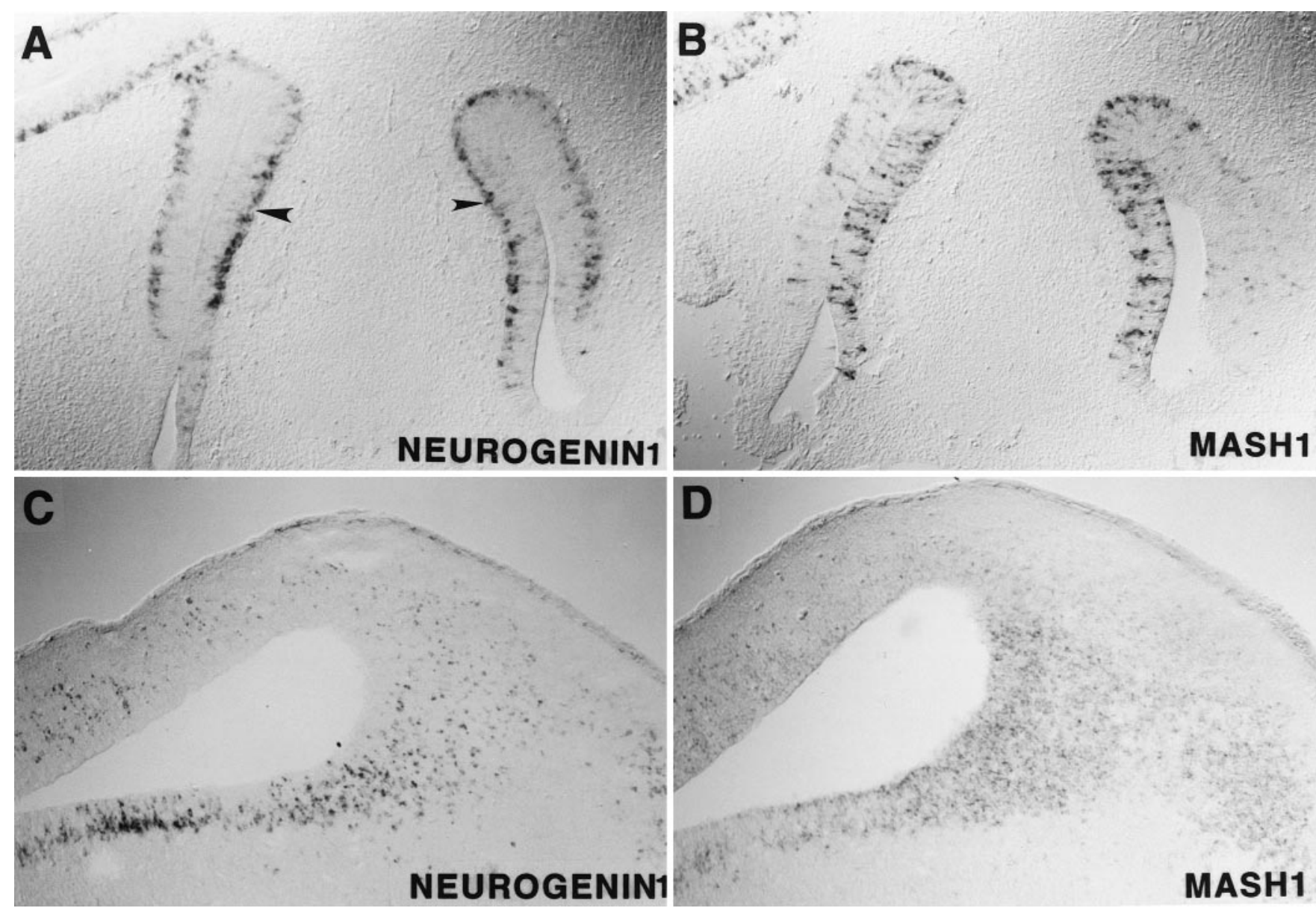

Figure 5. Overlapping but sequential expression of ngn1 and Mash1. Transverse sections through the olfactory epithelium $(A, B)$ and sagittal sections through the midbrain region $(C, D)$ of an E14.5 rat embryo are illustrated. ngn1 is expressed in cells located in the basal layer of the olfactory epithelium ( $A$, arrowhead), whereas Mash1 is expressed in cells spanning the epithelium (B). In the midbrain ngn1 expression is scattered $(C)$, whereas that of Mash1 is much more uniform $(D)$. Also, ngn1 expression (but not that of Mash1) is displaced laterally from the ventricular wall $(C, D)$.

in the spinal cord seems to extend to other regions of the CNS. For example, in the ventricular zone of the medulla and pons Jagged expression is restricted primarily to the region that also expresses high levels of $n g n 1$ (compare Fig. $6 E$ vs $F$ ). By contrast, in this brainstem region $D l l-1$ is expressed in a pattern that overlaps the expression domains of both Mash1 and ngn1 (compare Fig. $6 H$ vs $E, G$ ). Similarly, in the telencephalon strong Dll-1 expression is found in both the striatum, which expresses Mash1, and in the cortex, which expresses ngnl (Lindsell et al., 1996) (data not shown). By contrast, strong expression of Jagged in the brain is observed only in regions in which ngn1 (or ngn2; Gradwohl et al., 1996; Sommer et al., 1996) is strongly expressed, although faint expression can be seen in some regions that only express Mash1 (such as in the striatum). Thus, a generalization that emerges from these studies is that in the CNS Jagged expression is associated primarily with that of ngn1, whereas Dll-1 expression can be associated with that of either ngnl or of Mash1.

\section{DISCUSSION}

Mutual exclusivity of Mash1 and neurogenins expression during mammalian neurogenesis

The most striking finding to emerge from the present studies is that Mash1 and ngnl are expressed in complementary domains, which exhibit adjacent and nonoverlapping boundaries, in many regions of the CNS. In several regions of the CNS the comple- mentary domains delineated by Mash1 and ngh1 expression correspond to future functional subdivisions: in the spinal cord to the alar and basal regions and in the forebrain to the cortex and striatum. This mutual exclusivity mirrors the complementarity of expression of these bHLH genes in the PNS, in which Mash1 is expressed by autonomic and $n g n l$ by sensory progenitors.

Recently, we reported the isolation of two other ngn1-related genes, ngn 2 and ngn3 (Sommer et al., 1996). In the PNS ngn2 also is restricted to sensory lineages, such as in DRG and several epibranchial placode-derived ganglia in which Mash1 is not expressed (Sommer et al., 1996). In the CNS, particularly in the forebrain region, expression of ngn2 (also known as Math $4 A$; Gradwohl et al., 1996) shows a similar regional restriction like that of ngn1 (Sommer et al., 1996) and appears complementary to that of Mash1 (Gradwohl et al., 1996). By contrast, ngn3 is expressed only in extremely restricted regions of the CNS. For example, in the spinal cord $n g n 3$ expression is restricted to the region close to the floorplate (Sommer et al., 1996). Thus, much of the developing CNS can be divided into regions that express either Mash1 or one or more of the ngns.

In the PNS the complementary expression of Mash1 and the ngns appears to be reflected in the expression of several additional bHLH genes that may serve as potential downstream targets. Expression of NeuroD (Lee et al., 1995; Ma et al., 1996; Sommer et al., 1996) and NSCL1 (Begley et al., 1992) (Fig. 3C,D) is 

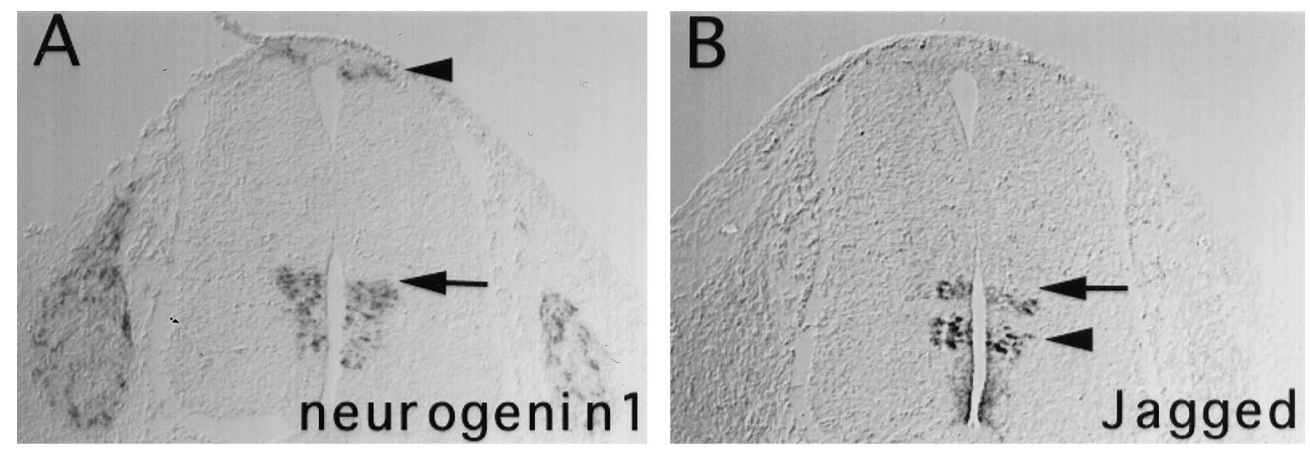

Figure 6. Comparisons of Mash1 and ngn1 expression to that of Dll-1 and Jagged. Adjacent transverse trunk sections of E13.5 $(A-D)$ and sagittal sections through the medulla and pons of E14.5 $(E-H)$ rat embryos are illustrated. The dorsal boundary of the Jagged stripe ( $B$, arrow) seems to correspond to the dorsal boundary of the ventral ngnl expression domain $(A$, arrow). The dorsal Dll-1 expression domain $(D)$ encompasses that of Mash1 (C) and extends both dorsally to the region expressing the dorsal ngnl stripe $(A$, arrowhead) and ventrally into the area corresponding to the top part of the ventral ngn1 stripe $(A)$. In the medulla ( $m d l)$ and pons (pons), complementarity between ngn 1 and Mash1 is indicated by the arrows ( $E$, $G)$. Expression of Jagged is restricted to the region in which $n g n 1$ is strongly expressed $(F)$, whereas Dll-1 is expressed throughout the ventricular zone $(H)$. Jagged expression in this region is discontinuous $(F$, arrowheads $)$, as in the spinal cord $(B)$.
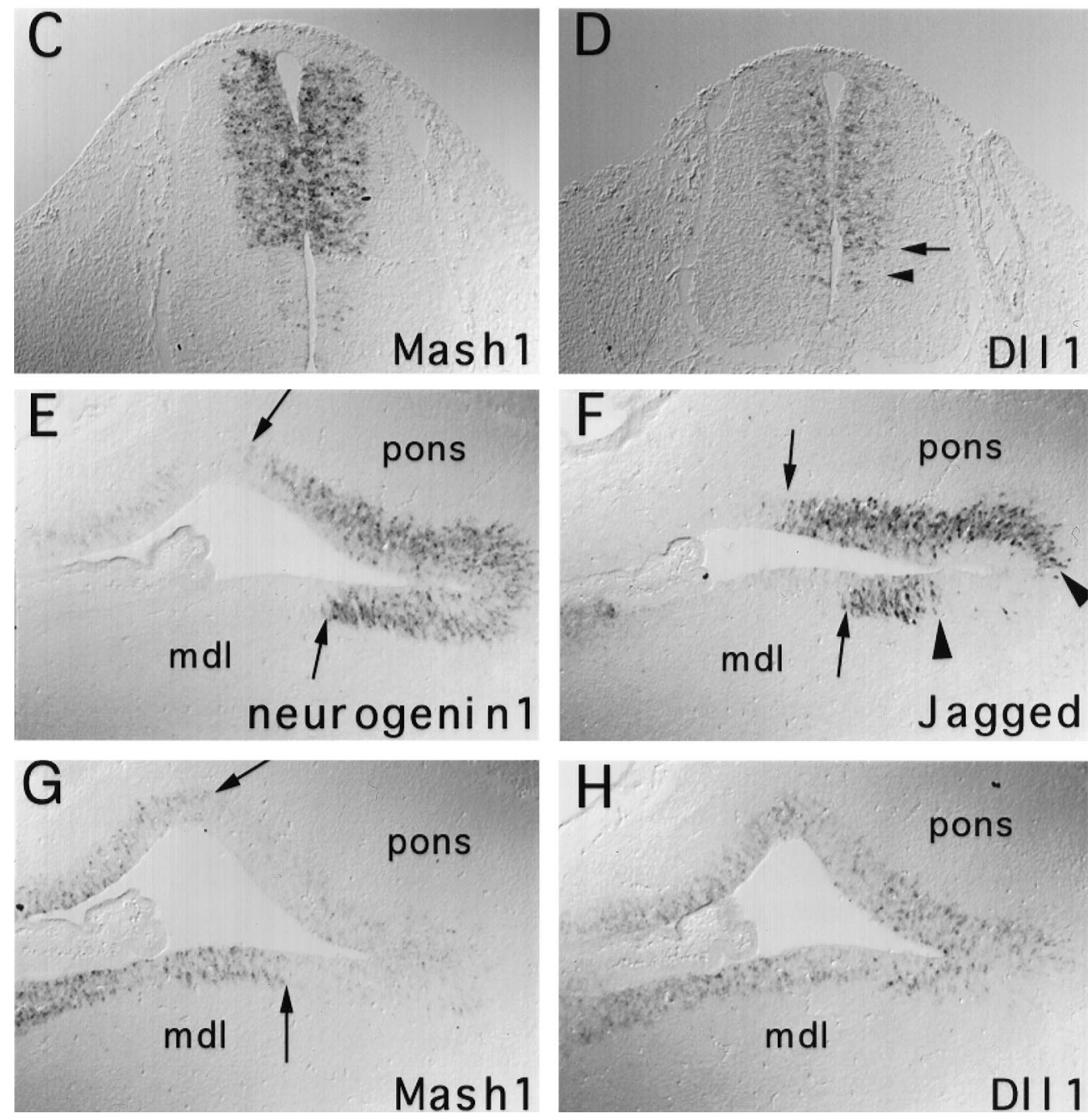

restricted to the sensory lineage, whereas $e H A N D$ expression is restricted to autonomic lineages (Cserjesi et al., 1995). The finding that Mash1 function is necessary for expression of $e H A N D$ in sympathetic ganglia indicates that eHAND is likely a direct or an indirect target of Mash1. In Xenopus a homolog of the ngns, $X$-ngnr-1, activates expression of $X$-NeuroD in a unidirectional cascade during primary neurogenesis (Ma et al., 1996). Similarly, $X$-NeuroD has been shown to activate expression of Xenopus NSCL (J. Lee, personal communication). Our in situ data (Fig. $2 A-D$; Sommer et al., 1996) are consistent with the idea that the ngns, neuroD, and NSCL1 may function in cascade during sensory neurogenesis in higher vertebrates, as well. Confirmation of this will, however, await loss-of-function analyses in ngn1 and/or ngn2 knock-out mice.

\section{Functional significance of complementary bHLH gene expression during neurogenesis}

Why are different classes of bHLH genes used to promote neurogenesis in different regions of the CNS and PNS? The answer to this question is likely to relate to differences in the functions of these genes. In the Drosophila PNS, atonal (which is structurally related to the ngns) is required for development of chordotonal organs, whereas achaete-scute is required for that of external sensory (es) organs (Jan and Jan, 1994). These genes are not functionally interchangeable, and recent data indicate that the key amino acid residues responsible for these functional differences lie in the basic region and likely mediate protein-protein rather than protein-DNA interactions (Chien et al., 1996). Thus in 
Drosophila structural differences among different neural bHLH proteins account for their ability to promote the determination of particular neural cell types, and the same is likely to be true in vertebrates. The noninterchangeable functions of different classes of neural bHLH proteins indicate that the choice of a neural (or neuronal) fate is coupled to the specification of a particular cell identity from the earliest developmental stages. However, a given bHLH gene is not sufficient to specify a particular neural cell type, because in both Drosophila and vertebrates individual bHLH genes are required for the development of more than one class of neurons. A single bHLH gene is, therefore, likely to act in combination with other region-specific transcriptional regulators in the pathways that determine neuronal identities. This would explain why, for example, expression of Mash1 is followed by that of eHAND in the PNS, but not the CNS.

\section{Overlapping expression of Mash1 and ngns}

Although spatial complementarity between expression of Mash1 and ngns seems to be the rule in most parts of the CNS, there are some regions in which these genes are coexpressed. For example, Mash1, ngn1, and ngn2 all are expressed in the midbrain region (Fig. 5C,D; data not shown). Other sites of ngn1 and Mash1 coexpression include the olfactory epithelium and midbrain. Interestingly, within these overlapping regions differences in the expression of these genes are clearly seen. For example, in the olfactory epithelium $n g n 1$ expression is restricted to the basal layer, whereas Mash1 expression spans the epithelium. Conversely, in the midbrain Mash1 is expressed in the ventricular zone, whereas both $n g n 1$ and $n g n 2$ are displaced slightly lateral to this region (Fig. 5C,D; data not shown). These differences suggest that even in these regions of overlap these genes may function at different developmental stages in the same cells or in distinct but intermingled groups of cells that develop on different schedules.

\section{Relationship of bHLH gene expression to that of Notch ligands}

Previously, it has been shown that the Notch ligands $c$-Delta-1 and c-Serrate-1 (a chick homolog of Jagged) and Delta-1 (Dll-1) and Jagged (Lindsell et al., 1996; Myat et al., 1996) exhibit complementary domains of expression in the spinal cord of chick and rat, respectively. In the chick this complementarity seems perfect. Our data indicate that it is partially lost in rat, however (Fig. 6). Because Jagged and Dll-1 are both ligands for Notch, they may be functionally equivalent. In that case, there would be little selection pressure to maintain such perfect complementarity. The bHLH genes Mash1 and ngn1 also exhibit complementary boundaries of expression in the rodent spinal cord. In the fly and the frog, homologs of these genes are demonstrably not functionally equivalent (Chien et al., 1996; Chitnis and Kintner, 1996; Ma et al., 1996). There may, therefore, be more selection pressure to maintain the complementarity of bHLH gene expression within the CNS. Analysis of the expression patterns of the chick homologs of these genes should be informative in this respect.

The side-by-side comparison of bHLH and Notch ligand gene expression presented here indicates that the boundaries of Jagged and Dll-1 expression do not correspond precisely to the boundaries between the domains of Mash1 and ngn1 expression (Figs. $4 B, C, 6)$. Specifically, Dll-1 is expressed in domains expressing either Mash1 or ngn1. On the other hand, high-level Jagged expression usually is (but not always) associated with that of ngn1. If, indeed, Jagged and Dll-1 are functionally equivalent, it is not clear why expression of Jagged should be associated preferentially with that of ngn1. This apparent linkage may reflect regulatory rather than functional constraints on the two genes.

The fact that the expression of the bHLH genes and that of the Notch ligands overlaps in the ventricular zone in both space and time raises the possibility that there is a regulatory interaction between these proteins in higher vertebrates, similar to that which occurs in Drosophila and Xenopus (Chitnis et al., 1995; Chitnis and Kintner, 1996; Ma et al., 1996) (for review, see Lewis, 1996). Specifically, proneural genes in the fly and X-ngnr-1 in the frog have been shown to activate expression of Delta and its Xenopus homolog, X-Delta-1, respectively. Our data are compatible with the idea that such an activation occurs in rodents as well, as suggested previously (Kunisch et al., 1994) (for review, see Lewis, 1996). Nevertheless, in Mash1 knock-out mice, expression of Dll-1 and Jagged in the CNS is not affected (our unpublished results), consistent with the failure to detect any other phenotypic defects in the CNS of these embryos (Guillemot et al., 1993). This may reflect functional redundancy or compensation between Mash1 and other unrelated bHLH genes; alternatively, Notch ligands may not be regulated by Mash1 in mice. Similarly, in the case of $n g n 1$ and ngn2, the overlap in their CNS expression may obscure defects in Notch ligand expression in single knock-out mice. Analysis of mice containing targeted mutations in both genes should, however, be informative.

\section{REFERENCES}

Akazawa C, Ishibashi M, Shimizu C, Nakanishi S, Kageyama R (1995) A mammalian helix-loop-helix factor structurally related to the product of Drosophila proneural gene atonal is a positive transcriptional regulator expressed in the developing nervous system. J Biol Chem 270:8730-8738

Begley CG, Lipkowitz S, Gobel V, Mahon KA, Bertness V, Green AR, Gough NM, Kirsch IR (1992) Molecular characterization of NSCL, a gene encoding a helix-loop-helix protein expressed in the developing nervous system. Proc Natl Acad Sci USA 89:38-42.

Bettenhausen B, Hrabe de Aggelis M, Simon D, Guenet JL, Gossler A (1995) Transient and restricted expression during mouse embryogenesis of Dll-1, a murine gene closely related to Drosophila Delta. Development (Camb) 121:2407-2418.

Brand M, Jarman AP, Jan LY, Jan Y-N (1993) asense is a Drosophila neural precursor gene and is capable of initiating sense organ formation. Development (Camb) 119:1-17.

Chien C-T, Hsia C-D, Jan LY, Jan Y-N (1996) Neuronal type information encoded in the basic helix-loop-helix domain of proneural genes. Proc Natl Acad Sci USA 93:13239-13244.

Chitnis A, Kintner C (1996) Sensitivity of proneural genes to lateral inhibition affects the pattern of primary neurons in Xenopus embryos. Development (Camb) 122:2295-2301.

Chitnis A, Henrique D, Lewis J, Ish-Horowicz D, Kintner C (1995) Primary neurogenesis in Xenopus embryos regulated by a homologue of the Drosophila neurogenic gene Delta. Nature 375:761-766.

Cross JC, Flannery ML, Blanar MA, Steingrimsson E, Jenkins NA, Copeland NG, Rutter WJ, Werb A (1995) Hxt encodes a basic helix-loophelix transcription factor that regulates trophoblast cell development. Development (Camb) 121:2513-2523.

Cserjesi P, Brown D, Lyons GE, Olson EN (1995) Expression of the novel basic helix-loop-helix gene $e H A N D$ in neural crest derivatives and extraembryonic membranes during mouse development. Dev Biol 170:664-678.

Ghysen A, Dambly-Chaudiere C, Jan LY, Jan Y-N (1993) Cell interactions and gene interactions in peripheral neurogenesis. Genes Dev 7:723-733.

Göbel V, Lipkowitz S, Kozak CA, Kirsch IR (1992) NSCL2, a basic domain helix-loop-helix gene expressed in early neurogenesis. Cell Growth Differ 3:143-148.

Gradwohl G, Fode C, Guillemot F (1996) Restricted expression of a novel murine atonal-related bHLH protein in undifferentiated neural precursors. Dev Biol 180:227-241.

Groves AK, George KM, Tissier-Seta J-P, Engel JD, Brunet JF, Anderson DJ (1995) Differential regulation of transcription factor gene expres- 
sion and phenotypic markers in developing sympathetic neurons. Development (Camb) 121:887-901.

Guillemot F, Joyner AL (1993) Dynamic expression of the murine achaete-scute homologue Mash1 in the developing nervous system. Mech Dev 42:171-185.

Guillemot F, Lo L-C, Johnson JE, Auerbach A, Anderson DJ, Joyner AL (1993) Mammalian achaete-scute homolog-1 is required for the early development of olfactory and autonomic neurons. Cell 75:463-476.

Hollenberg SM, Sternglanz R, Cheng PF, Weintraub H (1995) Identification of a new family of tissue-specific basic helix-loop-helix proteins with a two-hybrid system. Mol Cell Biol 15:3813-3822.

Jan Y-N, Jan LY (1993) HLH proteins, fly neurogenesis, and vertebrate myogenesis. Cell 75:827-830.

Jan Y-N, Jan LY (1994) Genetic control of cell fate specification in the Drosophila peripheral nervous system. Annu Rev Genet 28:373-393.

Jarman AP, Grau Y, Jan LY, Jan Y-N (1993) atonal is a proneural gene that directs chordotonal organ formation in the Drosophila peripheral nervous system. Cell 73:1307-1321.

Johnson JE, Birren SJ, Anderson DJ (1990) Two rat homologues of Drosophila achaete-scute specifically expressed in neuronal precursors. Nature 346:858-861.

Kunisch M, Haenlin M, Campos-Ortega JA (1994) Lateral inhibition mediated by the Drosophila neurogenic gene Delta is enhanced by proneural genes. Proc Natl Acad Sci USA 91:10139-10143.

Lee JE, Hollenberg SM, Snider L, Turner DL, Lipnick N, Weintraub H (1995) Conversion of Xenopus extoderm into neurons by NeuroD, a basic helix-loop-helix protein. Science 268:836-844.

Lewis J (1996) Neurogenic genes and vertebrate neurogenesis. Curr Opin Neurobiol 6:3-10.
Lindsell CE, Shawber CJ, Boulter J, Weinmaster G (1995) Jagged, a mammalian ligand that activates Notch1. Cell 80:909-917.

Lindsell CE, Boulter J, diSibio G, Gossler A, Weinmaster G (1996) Expression patterns of Jagged, Delta-1, Notch1, Notch2, and Notch3 genes identify ligand-receptor pairs that may function in neural development. Mol Cell Neurosci 8:14-27.

Lo L, Johnson JE, Wuenschell CW, Saito T, Anderson DJ (1991) Mammalian achaete-scute homolog 1 is transiently expressed by spatially restricted subsets of early neuroepithelial and neural crest cells. Genes Dev 5:1524-1537.

Ma Q, Kintner C, Anderson DJ (1996) Identification of neurogenin, a vertebrate neuronal determination gene. Cell 87:43-52.

Myat A, Henrique D, Ish-Horowicz D, Lewis J (1996) A chick homologue of Serrate and its relationship with Notch and Delta homologues during central neurogenesis. Dev Biol 174:233-247.

Porcher C, Swat W, Rockwell K, Fujiwara Y, Alt FW, Orkin SH (1996) The T cell leukemia oncoprotein SCL/tal-1 is essential for development of all hematopoietic lineages. Cell 86:47-57.

Rodriguez I, Hernandez R, Modolell J, Ruiz-Gomez M (1990) Competence to develop sensory organs is temporally and spatially regulated in Drosophila imaginal primordia. EMBO J 9:3583-3592.

Sommer L, Ma QF, Anderson DJ (1996) neurogenins, a novel family of atonal-related bHLH transcription factors, are putative mammalian neuronal determination genes that reveal progenitor cell heterogeneity in the developing CNS and PNS. Mol Cell Neurosci 8:221-241.

Sommer L, Shah N, Rao M, Anderson DJ (1995) The cellular function of MASH1 in autonomic neurogenesis. Neuron 15:1245-1258.

Weintraub H (1993) The MyoD family and myogenesis: redundancy, networks, and thresholds. Cell 75:1241-1244. 